\title{
Blood flow assessment at your fingertips
}

New research suggests that laser

Doppler imaging (LDI) could improve the understanding and management of peripheral vasculopathy in patients with SSc and Raynaud phenomenon. "LDI proved to be an objective and reproducible method for measurement of blood perfusion," reports

Cristiane Kayser, a senior author of the study, which compared LDI with nailfold capillaroscopy and fingertip lacticemy.

Baseline blood flow as measured by LDI was considerably lower in the fingers of 44 patients with Raynaud phenomenon secondary to SSc than in 40 healthy controls. In the former group the flow did not recover from the reduction caused by cold stimulus.

The severity of lactic acid build-up in the patients with SSc-measured by fingertip lacticemy-was indicative of the degree of the perfusion impairment; the lacticemy results correlated negatively with the LDI score. Intriguingly, however, the LDI results did not correlate with those of nailfold capillaroscopy, which identifies structural abnormalities in peripheral vessels. The authors suggest that LDI and nailfold capillaroscopy might assess independent aspects of microangiopathy.

The researchers plan to use LDI to monitor therapeutic responses in patients with SSc, and will investigate its use for assessing disease severity. Further studies planned will, Kayser adds, "help [to] understand the dissociation between morphologic and functional abnormalities in the microcirculation of SSc patients".

Emma Leah

Original article Correa, M. J. U. et al. Comparison of laser Doppler imaging, fingertip lacticemy test, and nailfold capillaroscopy for assessment of digital microcirculation in systemic sclerosis. Arthritis Res. Ther. 12, doi:10.1186/ar3112

Further reading Cutolo, M. et al. Assessing microvascular changes in systemic sclerosis diagnosis and management Nat. Rev. Rheumatol. 6, 578-587 (2010) 